\title{
STABLE AND UNSTABLE ROVIBRATIONAL STATES OF A MOLECULE
}

\author{
J. KONARSTK* \\ Department of Theoretical Chemistry, Faculty of Chemistry \\ A. Mickiewicz University of Poznań, Grunwaldzka 6, 60-780 Poznań, Poland
}

(Received April 1, 1999; in final form May 18, 1999)

Stability of motions for different potential functions describing vibrational motions of rotating molecule has been discussed. It was shown that an equilibrium position for nonrotating $r_{0}$ and rotating $r_{J}$ molecules for almost all the examined potentials is a node, but for the molecule described by the soft body model a critical value of rotation quantum number $J_{c}$ occurs above which $r_{0}$ is a saddle point, and the stable motion may occur only around $r_{J}$.

PACS numbers: 33.15.-e, 33.15.Dj, 33.15.Mt

Stability or instability of motions plays an important role in many problems of physics, astronomy, molecular physics and is determined by the form of potential used to describe the problem considered [1-9].

In this paper I would like to discuss this problem for rotational-vibrational (rovibrational) motions which are described by many different potential functions (see for example $[10,11]$ ). For this purpose eigenvalue $\lambda$ of stability matrix $A$ will be calculated for two fixed points $r_{0}$ and $r_{J}[1,5,7]$. When $\lambda$ is a real and positive number, the fixed point is a repeller, whereas for an imaginary one, the motion examined is stable around this point which is a centre.

The elements of the stability matrix $A_{i j}$ are calculated by the following method $[1,2,5,7]$. Let us consider a motion described by the Hamiltonian

$$
H=\sum_{i=1}^{n} \frac{p_{i}^{2}}{2 m_{i}}+V\left(q_{1}, \ldots, q_{n}\right)
$$

where $p_{i}$ is the generalized momentum for the $i$-th degree of freedom $(i=1, \ldots, f)$, $q_{i}$ is the generalized coordinate for the $i$-th independent motion $(i=1, \ldots, f)$. Now

$$
A_{k l}=\left(\frac{\partial F_{k}}{\partial x_{l}}\right)_{q_{i}^{0}}, \quad k=1, \ldots, f, l=1, \ldots, f
$$

\footnotetext{
*e-mail: konarski@ROVIB.amu.edu.pl
} 
and for $k=1, \ldots, f$

$$
F_{k}=\frac{\partial H}{\partial p_{n}}, \quad n=1, \ldots, f, \quad F_{f+n}=-\frac{\partial H}{\partial q_{n}},
$$

$x_{1}, \ldots, x_{f}=q_{1}, \ldots, q_{f}, x_{f+1}, \ldots, x_{2 f}=p_{1}, \ldots, p_{f} ; q_{i}^{0}$ is the $i$-th fixed point.

The eigenvalues of the stability matrix are calculated from the equation

$\operatorname{Det}\left|A_{k l}-\lambda \delta_{k l}\right|=0$.

Let us consider an example as simple as possible, i.e. a one-dimensional harmonic oscillator with centrifugal force, now the Hamiltonian has the form

$$
H=\frac{p^{2}}{2 \mu}+\frac{1}{2} k r^{2}+\frac{R^{2}}{2 \mu r^{2}}
$$

and $F_{1}=p / \mu, F_{2}=-k r+R^{2} /\left(\mu r^{3}\right)$, the fixed point is obtained from the condition $F_{2}=0$, and is equal to $r_{0}^{4}=R^{2} / \mu k$, now

$$
\begin{aligned}
& A_{11}=\frac{\partial F_{1}}{\partial r^{r}}=0, \quad A_{12}=\frac{\partial F_{1}}{\partial p}=\frac{1}{\mu}, \\
& A_{21}=\left(\frac{\partial F_{2}}{\partial r^{2}}\right)_{r_{0}}=-k-\frac{3 R^{2}}{\mu \cdot r_{0}^{4}}=-4 k, \quad A_{22}=0 .
\end{aligned}
$$

The eigenvalue of problem for the case has the form

$$
\left|\begin{array}{cc}
-\lambda, & 1 / \mu \\
-4 k, & -\lambda
\end{array}\right|=0, \text { so } \lambda^{2}+\frac{4 k}{\mu}=0 \text { and } \lambda=i \sqrt{\frac{4 k}{\mu}},
$$

has an imaginary value for $r_{0}$ so the fixed point is a node $[1,7]$.

Table I shows the results obtained for different potentials, as well as the role of rotation of the body considered in the destabilization or stabilization of motion. These results have been calculated for diatomic molecules, because for these species the potential which properly describes the internal motion is well known [10-12]. For these calculations the fixed point $r_{0}$ was obtained from the condition $\partial V / \partial r=0$, whereas the second fixed point $r_{J}$ was obtained from the overall equilibrium condition, i.e from the following equation:

$$
-\frac{\partial V}{\partial r}+\frac{R^{3}}{\mu r^{3}}=0
$$

where $R^{2} / \mu r^{3}$ describes a force brought a.bout by rotation of a molecule.

As follows from Table I the rotational motion of a molecule stabilizes the nodal feature of the fixed points $r_{0}$ and $r_{J}$. Interesting results have been obtained for a double minimum potential (see no. 5 in Table I) for which rotation of a molecule stabilizes $r_{0}$ (the point for which the potential has a maximum).

In a series of papers [13-19] it has been shown that the detected rovibrational transitions are very well assigned within the soft or deformable model, i.e. within the model which takes into consideration a deformation of a molecule brought about by its rotation. This model introduces the references configuration, i.e the rigid configuration around which the nuclei perform their motions, from the condition

$$
f_{i}=-\frac{\partial V}{\partial r}+m_{i} \omega \times \omega \times r_{i}
$$


TABLE I

Values of the stability matrix for different potentials.

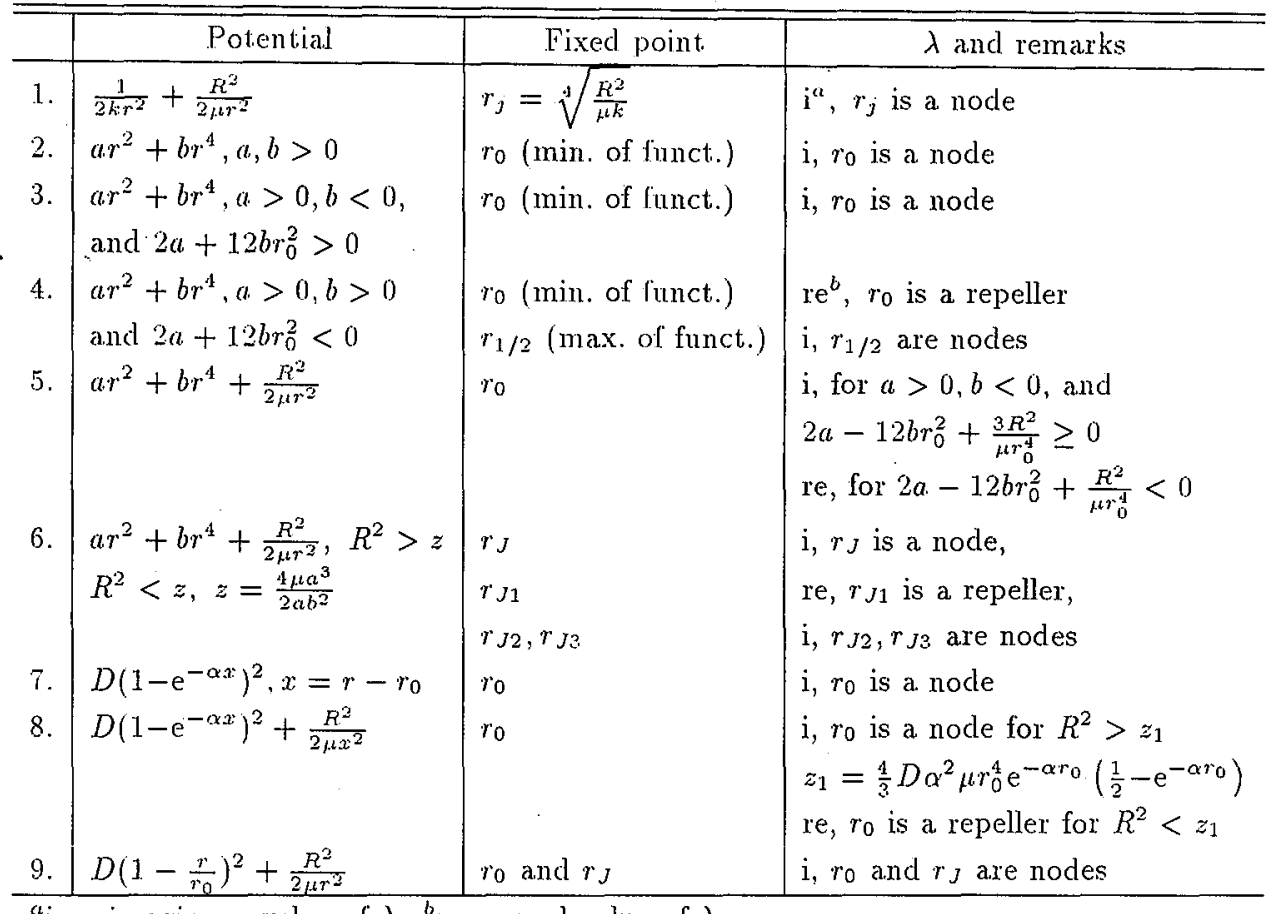

a $\mathrm{i}$ - imaginary value of $\lambda,{ }^{b}$ re - real value of $\lambda$.

where $f_{i}^{0}=-\partial V / \partial r$ is a force acting upon the $i$-th atom of a molecule brought about by electronic structure of a molecule, and $\omega$ is the angular velocity of rotation of a molecular coordinate system relative to the laboratory one.

The solution of this equation, $r_{i J}$, gives the equilibria positions which depend on the angular velocity $\omega$. As a result the overall angular momentum is given by the equation

$$
P_{\alpha}=\frac{\partial L}{\partial \omega_{\alpha}}=\sum\left[m_{i}\left(r_{i} \times \omega \times r_{i}\right)_{\alpha}+m_{i}\left(r_{i} \times v_{i}\right)_{\alpha}+\left(\partial r_{J i} / \partial \omega_{\alpha}\right) \nabla_{i} L\right]
$$

so $P_{c}=R_{\alpha}+K_{\alpha}, \alpha=x, y, z$, where

$$
K_{\alpha}=\sum_{i} \frac{\partial v^{\prime} J i}{\partial \omega_{\alpha}} \nabla_{i} L
$$

is an additional angular momentum brought about by the changing of the distance of the $i$-th element of the soft body from the equilibrium position $r_{i J}$ due to vibrations (for: $r=r_{i J}, \nabla_{i} L=0$ and $K_{\alpha}=0$, see Eq. (1))[13].

Fol diatomic molecules

$$
R=\mu r^{2} \omega=P-K
$$

and the Hamiltonian for internal motions has the following form $[13,14]$ :

$$
H=\frac{p^{2}}{2 \mu}+\frac{\dot{P}^{2}-K^{2}}{2 \mu r^{2}}+V(r) \text {. }
$$


From this equation we see that internal motions of the soft body are described by the effective potential

$$
V_{\mathrm{ef}}=V(r)+\frac{P^{2}-K^{2}}{2 \mu r^{2}}
$$

The additional angular momentum is calculated from Eq. (2), i.e from the equation

$$
K=s\left[-\frac{\partial V}{\partial r}+\frac{(P-K)^{2}}{\mu r^{3}}\right] \text {, }
$$

where $s^{2}=\left(\partial r_{j} / \partial \omega_{x}\right)^{2}+\left(\partial r_{j} / \partial \omega_{y}\right)^{2}$. The solution of this equation leads to the following formula for additional angular momentum $K$ :

$$
K=\frac{\mu r^{3}+A-d}{\kappa},
$$

where $A=4 B_{0} J(J+1) \mu r_{0} r_{J}^{2} / D, B_{0}=h^{2} /\left(2 \mu r_{0}^{2}\right), \kappa=\sqrt{8 B_{0} J(J+1) \mu r_{J}^{4} / D^{2}}$, $d=\sqrt{\mu^{2} r^{6}+2 A \mu r^{3}+4 A \mu r_{J}^{2}\left(r-r_{0}\right)}$ and $r_{J}$ is obtained from Eq. (1) where the Fues-Kratzer $[20,21]$ potential has been applied for clescription of the internal motion. As a result $r_{J}=r_{0}\left[1+B_{0} J(J+1) / D\right]$, and $D$ is a constant related to the dissociation energy of a. molecule.

\section{TABLE II}

\begin{tabular}{|c|c|c|c|}
\hline$J$ & \multicolumn{2}{|c|}{$\lambda$} & remarks \\
\hline & $r_{0}$ & $r_{J 1}$ & \\
\hline 1 & imaginary & ima.ginary & $r_{0}$ and $r_{J}$ are nodes \\
\hline 112 & imaginary & imaginary & $r_{0}$ and $r_{J}$ are nodes \\
\hline 113 & real & imaginary & $\begin{array}{l}r_{0} \text { is a saddle point, } \\
r_{J} \text { is a node }\end{array}$ \\
\hline 120 & real & imaginary & $\begin{array}{l}r_{0} \text { is a saddle point, } \\
r_{J} \text { is a node }\end{array}$ \\
\hline
\end{tabular}

Eigenvalue of the stability matrix for $\mathrm{Ar}_{2}\left({ }^{1} \sum_{\mathrm{g}}^{+}\right)$calculated for two fixed points $r_{0}$ and $r_{J}$.

\section{TABLE III}

Dependence of the critical rotational quantum number $J_{\mathrm{c}}$ on $Y=B_{0} / D$.

\begin{tabular}{c|c|c|r}
\hline$Y$ & $r_{0}$ & $r_{J}$ & \multicolumn{1}{|c}{$J_{c}$} \\
\hline $1.349 \times 10^{-2}$ & saddle point & node & 4 \\
$1.336 \times 10^{-3}$ & saddle point & node & 11 \\
$1.9046 \times 10^{-5}$ & saddle point & node & 113
\end{tabular}

Table II presents the results obtained for $\operatorname{Ar}_{2}\left({ }^{1} \sum_{\mathrm{g}}^{+}\left(0_{\mathrm{g}}^{+}\right)\right)$molecule (the $r_{0}$ and $D$ parameters used in these calculations have been taken from Ref. [13]). Table III shows the dependence of eigenvalue of the stability matrix $\lambda$ on $Y$ parameter 
defined by the relation $Y=B_{0} / D$, and gives critical values of rotational quantum number $J_{c}$ at which $r_{0}$ changes its character from a node to a saddle point. drawn:

From the above presented calculations the following conclusions may be

(1) for all the widely applied potentials, namely for the harmonic and nonharmonic ones, the two fixed points, $r_{0}$ and $r_{J}$, are nodes, and the motions around these points are stable,

(2) rotational motions preserve the character of $r_{0}$ for higher energies, i.e rotation stabilizes the structure of a molecule (this is the well-known gyroscopic effect (see for example [5])),

(3) for the soft body model the character of the fixed points depends on rotational quantum number $J$. Below some $J$ called the critical rotation quantum number $J_{c}$ both fixed points, namely $r_{0}$ and $r_{J}$, are nodes, whereas above $J_{\mathrm{c}}$ the motion around $r_{0}$ is unstable ( $r_{0}$ is a saddle point) but $r_{J}$ is still a node, so a motion around this point is stable. As a consequence a molecule changes its shape above the critical value of $J$.

\section{References}

[1] V.I. Arnold, Mathematical Methods in Classical Mechanics, Springer Verlag, New York 1978.

[2] R.I. Devaney, An Introduction to Chaotic Dynamical System, 2nd ed., Benjamin/Cummings, Menlo Park 1986.

[3]. M.S. Child,. Semiclassical Mechanics with Molecular Applications, Claredon Press, Oxford 1991.

[4] J. Wisclom, Nucl. Phys. B, Proc. Suppl. 2, 391 (1987).

[5] F. Scheck, Mechanics, Springer Verlag: Berlin 1990.

[6] Chaos and Quantum Physics, Les Hauches Lecture 1989, Eds. M.J. Giannoni, A. Voros, J. Zinn-Justin, session LII, North-Holland, Amsterdam 1991.

[7] R.C. Hilborn, Chaos and Non-linear Dynamics, Oxford University Press, Oxford 1994.

[8] J. Cizek, J. Paldus, J. Chem. Phys, 53, 825 (1970).

[9] J. Paldus, J. Cizek, J. Polym. Sci. Part C 29, 199 (1970).

[10] V.P. Varshni, Rev. Mod. Phys. 29, 644 (1957).

[11] G. Simons, J.M. Finlan, Phys, Rev. Lett. 33, 131 (1974).

[12] E.S. Kryachko, T. Koga, Adv. Quantum Chem. 17, 98 (1985).

[13] M. Molski, J. Konarski, Phys. Rev. A 47, 711 (1993).

[14] J. Konarski, Int. J. Quant. Chem. 51, 439 (1994).

[15] M. Molski, J. Konarski, Canad. J. Phys. 73, 59 (1995).

[16] M. Molski, J. Mol. Spectrosc. 182, 1 (1997).

[17] M. Molski, J. Mol. Spectrosc. 185, 256 (1997).

[18] M. Molski, J. Konarski, Polish J. Chem. (special issue) 72, 1398 (1998).

[19] J.F. Ogilvie, M. Molski, Spectrochim. Acta A, in print.

[20] E. Fues, Ann. Phys. (Paris) 80, 367 (1926); ibid. 81, 281 (1926).

[21] A. Kratzer, Z. Phys. 3, 289 (1920). 